\title{
A Constructive Examination of Rectifiability
}

\author{
DOUGLAS S. BRIDGES \\ MATTHEW HENDTLASS \\ ERIK PALMGREN
}

\begin{abstract}
We present a Brouwerian example showing that the classical statement 'Every Lipschitz mapping $f:[0,1] \rightarrow[0,1]$ has rectifiable graph' is essentially nonconstructive. We turn this Brouwerian example into an explicit recursive example of a Lipschitz function on $[0,1]$ that is not rectifiable. Then we deal with the connections, if any, between the properties of rectifiability and having a variation. We show that the former property implies the latter, but the statement 'Every continuous, real-valued function on $[0,1]$ that has a variation is rectifiable' is essentially nonconstructive.
\end{abstract}

2010 Mathematics Subject Classification 03F60, 26A16, 26A99 (primary)

Keywords: Constructive/recursive analysis, Lipschitz, rectifiable, Brouwerian example, recursive counterexample.

\section{Introduction}

Consider a real-valued function $f$ on a closed interval $[a, b]$. If $P: a=x_{0} \leqslant x_{1} \leqslant$ $\cdots \leqslant x_{n}=b$ is a partition of $[a, b]$, then the corresponding polygonal approximation to $f$ has length

$$
l_{f, P} \equiv \sum_{i=1}^{n-1} \sqrt{\left(x_{i+1}-x_{i}\right)^{2}+\left(f\left(x_{i+1}\right)-f\left(x_{i}\right)\right)^{2}} .
$$

We say that $f$

- has bounded length if there exists $c>0$ such that $l_{f, P} \leqslant c$ for each partition $P$ of $[a, b]$;

- is rectifiable if its length,

$$
\sup \left\{l_{f, P}: P \text { is a partition of }[a, b]\right\},
$$

exists; 
- is Lipschitz if there exists $\kappa>0$ such that $|f(x)-f(y)| \leqslant \kappa|x-y|$ for all $x, y \in[a, b]$.

Every Lipschitz function has bounded length: for with $f, \kappa$, and $P$ as above,

$$
l_{f, P} \leqslant(1+\kappa) \sum_{i=1}^{n-1}\left(x_{i+1}-x_{i}\right)=1+\kappa .
$$

The classical least-upper-bound principle ensures that if $f$ has bounded length, then it is rectifiable. But in the constructive context of this paper, that principle implies the law of excluded middle (LEM) and so is inadmissible. The constructive least-upper-bound principle requires the additional hypothesis that the set $S \subset \mathbf{R}$ whose supremum is sought must be not only inhabited and bounded above, but also upper-order-located, in the sense that whenever $\alpha<\beta$, either $x \leqslant \beta$ for all $x \in S$ or else there exists (we can find) $x \in S$ with $\alpha<x$ (see Bishop and Bridges [4, Page 37] or Bridges and Vîţă [9, Theorem 2.1.18]).

With the aid of Specker's theorem [17] it is not hard to produce a recursive example of a pointwise, but not uniformly continuous function $f:[0,1] \rightarrow \mathbf{R}$ that has bounded length but is not rectifiable. The motivation for this paper lies in the question: Is every Lipschitz function $f:[0,1] \rightarrow \mathbf{R}$ constructively rectifiable? Our first main result (Proposition 2) gives a Brouwerian example showing that the rectifiability of all real-valued Lipschitz functions-and hence of all real-valued, uniformly continuous ones-on [0,1] implies the essentially nonconstructive limited principle of omniscience:

LPO: For each binary sequence $\left(a_{n}\right)_{n \geqslant 1}$, either $a_{n}=0$ for all $n$ or else there exists $n$ such that $a_{n}=1$.

This leads to our second main result (Theorem 7), providing an explicit example of a recursive Lipschitz function that has bounded length but is not rectifiable. The proof of the latter depends on a lemma of interest in its own right (Lemma 5), which enables us to pass from rectifiability over the whole interval $[0,1]$ to rectifiability over each of its compact subintervals.

In the second part of the paper we consider the possibility of connecting rectifiability with the property of having a variation (which, classically, reduces to that of bounded variation). In particular, we show that rectifiable continuous functions on $[0,1]$ have a variation, but the converse implies LPO (Corollary 9).

The constructive framework, BISH, of our work is that of Bishop [3, 4, 9] (see also Troelstra and van Dalen [18]), in which the logic is intuitionistic and we adopt a 
mathematical foundation such as the set theories CZF (Aczel and Rathjen [1, 2]) and CMST (Bridges and Alps [6]), or Martin-Löf's type theory (Martin-Löf [14, 15], Nordström, Peterson and Smith [16]). One model (in a purely informal sense) or interpretation of BISH is the recursive one, RUSS, which can be regarded as BISH plus the Church-Markov-Turing thesis and, if desired, Markov's principle of unbounded search (see Kushner [12], Markov [13] or Bridges and Richman [8, Chapter 3]); that model is the setting for Theorem 7 .

\section{Lipschitz curves need not be rectifiable}

We begin our technical presentation with a lemma.

Lemma 1 Let $\left(a_{n}\right)_{n \geqslant 1}$ be an increasing binary sequence with $a_{1}=0$, and let $b>0$. Then there exists a Lipschitz function $f:[0, b] \rightarrow[0, b]$ such that

(i) $f(0)=f(b)=0$;

(ii) $f$ has Lipschitz constant 2;

(iii) if $f$ is rectifiable, then either $a_{n}=0$ for all $n$ or there exists $n$ with $a_{n}=1$; and

(iv) if $f$ is differentiable at any point of $[0, b]$, then either $a_{n}=0$ for all $n$ or there exists $n$ with $a_{n}=1$.

Proof Define a sequence $\left(f_{n}\right)_{n \geqslant 1}$ of continuous spike functions from $[0, b]$ into [0,1] as follows. If $a_{n}=0$ or $a_{n-1}=1$, set $f_{n} \equiv 0$. If $a_{n}=1-a_{n-1}$, construct $f_{n}$ so that

- it is uniformly continuous on $[0, b]$,

- for $i \in\left\{0,1, \ldots, 2^{n}-1,2^{n}\right\}, f_{n}\left(i 2^{-n} b\right)=0$,

$$
f_{n}\left(\left(i+\frac{1}{2}\right) 2^{-n} b\right)=\frac{b}{2^{n}+1},
$$

and $f_{n}$ is linear on each half of the interval $\left[i 2^{-n} b,(i+1) 2^{-n} b\right]$.

Note that if $a_{n}=0$ for all $n$, then $f_{n}=0$; whereas if $a_{n}=1-a_{n-1}$, then the length of the spiked path $f_{n}$ joining 0 to $b$ is

$$
>2 b \underbrace{\left(\frac{1}{2^{n}+1}+\frac{1}{2^{n}+1}+\cdots+\frac{1}{2^{n}+1}\right)}_{2^{n} \text { terms }}=\frac{2^{n+1} b}{2^{n}+1} \geqslant \frac{8}{5} b
$$


(the last inequality following because $n \geqslant 2$ ). Note also that if $a_{n}=1-a_{n-1}$, then the absolute value of the slope of the spikes of $f_{n}$ is

$$
\frac{f\left(2^{-n-1} b\right)-f(0)}{2^{-n-1} b-0}=\frac{2^{n+1}}{b} \times \frac{b}{2^{n}+1}=\frac{2^{n+1}}{2^{n}+1} .
$$

which is less than 2 (and, incidentally, increases to the limit 2 as $n \rightarrow \infty$ ). Hence $f_{n}$ is Lipschitz, with Lipschitz constant 2.

Since $\left\|f_{n}\right\| \leqslant 2^{-n} b$ for each $n$, the series $\sum_{n=1}^{\infty}\left\|f_{n}\right\|$ converges, so $\sum_{n=1}^{\infty} f_{n}$ converges in norm to a uniformly continuous function $f:[0, b] \rightarrow \mathbf{R}$, with (as is clear) $f(0)=$ $f(b)=0$. For each $x \in[0, b]$, either $f(x)>0$ or $f(x)<2$; in the former case, there exists exactly one $n$ such that $f(x)=f_{n}(x)$, so $\|f\| \leqslant 2$. It then follows that $f$ is Lipschitz, with Lipschitz constant 2, and (see above) that the length of the curve $y=f(x)$ is $>b$.

Now suppose that the curve $y=f(x)$ is rectifiable, with length $s$. Either $s>b$ or else $s<8 b / 5$. In the first case we can find $x \in[0,1]$ with $f(x)>0$, and hence $n$ with $a_{n}=1$. In the second case we must have $a_{n}=0$ for all $n$. Thus (iii) holds.

To deal with (iv), suppose, initially, that $f$ is differentiable at the point $k 2^{-N} b$, where $N \in \mathbf{N}$ and $0 \leqslant k \leqslant 2^{N}$. We may further suppose that $a_{N}=0$. If there exists $n>N$ such that $a_{n}=1-a_{n-1}$, then $f=f_{n}$ and $k 2^{-N} b=\left(k 2^{n-N}\right) 2^{-n} b$ is a point where two adjacent spikes of $f_{n}$ meet; whence $f$ is not differentiable at $k 2^{-N} b$, a contradiction. Thus $a_{n}=0$ for all $n>N$ and therefore for all $n$. Now let $x$ be any point of $[0, b]$, and suppose that $f^{\prime}(x)$ exists. Either $\left|f^{\prime}(x)\right|>0$ or $\left|f^{\prime}(x)\right|<1$. In the first case there exists $h \neq 0$ such that $f(x+h) \neq f(x)$; so either $f(x+h) \neq 0$ or $f(x) \neq 0$. Taking, for example, the case where $f(x+h) \neq 0$, compute $\nu$ such that $\sum_{n=1}^{\nu} f_{n}(x+h) \neq 0$. Then there exists $n \leqslant \nu$ such that $f_{n}(x+h) \neq 0$ and therefore $a_{n}=1$. On the other hand, in the case where $\left|f^{\prime}(x)\right|<1$, if there exists $n$ with $a_{n}=1-a_{n-1}$, then, in view of the foregoing observation, $x$ cannot lie on any open segment of a side of any spike of $f$, and so must be one of the three vertices of a spike. This is absurd, since we have just proved that $f$ is not differentiable at such a vertex. Hence in this case we must have $a_{n}=0$ for all $n$. This completes the proof of (iv).

Lemma 1 immediately provides us with two interesting Brouwerian counterexamples for Lipschitz curves:

Proposition 2 The statement 'Every real-valued Lipschitz function on [0,1] is rectifiable' implies LPO. 
Proposition 3 The statement 'Every Lipschitz function on [0,1] is differentiable at some point' implies LPO.

In this context, the following is worth noting.

Proposition 4 LPO implies that every sequentially continuous function $f:[0,1] \rightarrow \mathbf{R}$ with bounded length is rectifiable.

Proof Let $f:[0,1] \rightarrow \mathbf{R}$ be sequentially continuous and have bounded length, and let $\left(P_{n}\right)_{n \geqslant 1}$ be an enumeration of the partitions of $[0,1]$ with rational endpoints. Given real numbers $\alpha, \beta$ with $\alpha<\beta$, and using countable choice, construct a binary sequence $\left(\lambda_{n}\right)_{n \geqslant 1}$ such that if $\lambda_{n}=0$, then $l_{f, P_{n}}<\beta$, and if $\lambda_{n}=1$, then $l_{f, P_{n}}>\alpha$. Applying LPO, we see that either $\lambda_{n}=0$ for all $n$ or else there exists $N$ such that $\lambda_{N}=1$. In the second case, $l_{f} \geqslant l_{f, P_{N}}>\alpha$. In the first case, suppose that there exists a partition $P$ of $[0,1]$ such that $l_{f, P}>\beta$. Since $f$ is sequentially continuous, we can find such a partition $P$ with rational endpoints. Then $P=P_{\nu}$ for some $\nu$, so $l_{f, P_{\nu}}>\beta$ and therefore $\lambda_{\nu}=1$. This contradiction ensures that $l_{f, P} \leqslant \beta$ for all partitions $P$ of $[0,1]$. Since $f$ has bounded length, its rectifiability now follows from the constructive least-upper-bound principle.

In the next section we convert these Brouwerian examples into a full-blooded counterexample in the recursive setting.

\section{A recursive counterexample}

To produce the promised recursive counterexample, we develop a general lemma, whose proof is derived from that of the particular application to functions of bounded variation (Bridges [5, Theorem 3]).

By a pseudoquasimetric on a set $X$ we mean a mapping $d: X \times X \rightarrow \mathbf{R}$ such that for all $x, y, z$ in $X$,

- $d(x, y) \geqslant 0$ and $d(x, x)=0$

- $d(x, y) \geqslant d(x, z)+d(z, y)$.

A continuous pseudoquasimetric on a metric space $(X, \rho)$ is a pseudoquasimetric that is uniformly continuous as a mapping from $X \times X$, taken with the product metric induced by $\rho$, into $\mathbf{R}$. 
Lemma 5 Let $I=[0,1]$, and let $d: I \times I \rightarrow \mathbf{R}$ be a continuous pseudoquasimetric on $I$. For each compact interval $[a, b] \subset I$ and each partition $P: a=x_{1} \leqslant x_{2} \leqslant \cdots \leqslant x_{n}=b$ of $[a, b]$, write

$$
\sum_{d, P} \equiv \sum_{i=0}^{n-1} d\left(x_{i}, x_{i+1}\right)
$$

Suppose that

$$
s \equiv \sup \left\{\sum_{d, P}: P \text { is a partition of } I\right\}
$$

exists. Then for each compact subinterval $J$ of $I$,

$$
\sup \left\{\sum_{d, Q}: Q \text { is a partition of } J\right\}
$$

exists.

Proof Fix a compact subinterval $J \equiv[a, b]$ of $[0,1]$ with rational endpoints $a, b$. Let $0<\alpha<\beta$, and $\varepsilon=\frac{1}{3}(\beta-\alpha)$. Since $d$ is uniformly continuous, we can construct a partition $P: 0=x_{0}<x_{1}<\cdots<x_{N}=1$ consisting of distinct rational points of $I$ such that $\sum_{d, P}>s-\varepsilon$. Since $a$ and the $x_{i}$ are rational, there exists $p$ such that $x_{p}<a \leqslant x_{p+1}$. Since $d$ satisfies the triangle inequality, adding $a$ to the partition $P$ does not decrease the value of $\sum_{d, P}$; we may therefore assume that $a=x_{m}$, and likewise that $b=x_{m+k}$, for some $m$ and $k$. Letting

$$
t \equiv \sum_{i=m}^{m+k-1} d\left(x_{i}, x_{i+1}\right),
$$

we have either $t>\alpha$ or $t<\alpha+\varepsilon$. In the latter case, suppose that there exists a partition $a=y_{0} \leqslant y_{1} \leqslant \cdots \leqslant y_{\nu}=b$ such that

$$
\sum_{j=0}^{\nu-1} d\left(y_{j}, y_{j+1}\right)>\beta-\varepsilon .
$$

Set $z_{i}=x_{i}$ if $0 \leqslant i \leqslant m, z_{i}=y_{i-m}$ if $m<i \leqslant m+\nu$, and $z_{i}=x_{i-\nu+k}$ if $m+\nu<i \leqslant N+\nu-k$. Then

$$
\begin{aligned}
\sum_{i=1}^{N+\nu-k-1} d\left(z_{i}, z_{i+1}\right) & =\sum_{i=0}^{N-1} d\left(x_{i}, x_{i+1}\right)-t+\sum_{j=0}^{\nu-1} d\left(y_{j}, y_{j+1}\right) \\
& >(s-\varepsilon)-(\alpha+\varepsilon)+(\beta-\varepsilon)=s+(\beta-\alpha)-3 \varepsilon>s,
\end{aligned}
$$

which contradicts the definition of $s$. It follows that if $t<\alpha+\varepsilon$, then $\sum_{d, Q}<\beta$ for each partition $Q$ of $[a, b]$. We have therefore shown that either there exists a partition 
$Q$ of $J$ with $\sum_{d, Q}>\alpha$ or else $\sum_{d, Q}<\beta$ for all partitions $Q$ of $J$. Since $\alpha, \beta$ are arbitrary positive numbers with $\alpha<\beta$, the constructive least-upper-bound principle now ensures that the desired supremum exists. Finally, the continuity of $d$ enables us to remove the restriction that $a$ and $b$ be rational.

Proposition 6 If $f:[0 ; 1] \rightarrow \mathbf{R}$ is uniformly continuous and rectifiable, then the restriction of $f$ to any compact subinterval of $[0,1]$ is rectifiable.

Proof Take

$$
d\left(x_{1}, x_{2}\right) \equiv \sqrt{\left(x_{1}-x_{2}\right)^{2}+\left(f\left(x_{1}\right)-f\left(x_{2}\right)\right)^{2}} \quad\left(x_{1}, x_{2} \in[0,1]\right)
$$

in Lemma 4.

Another application of Lemma 5 arises in connection with a uniformly continuous function $f:[0,1] \rightarrow \mathbf{R}$ of bounded variation. For each partition $P: 0=x_{0} \leqslant x_{1} \leqslant$ $\cdots \leqslant x_{n}=1$ define

$$
\begin{aligned}
& v_{f, P}^{+} \equiv \sum_{i=0}^{n-1} \max \left\{0, f\left(x_{i+1}\right)-f\left(x_{i}\right)\right\}, \\
& v_{f, P}^{-} \equiv-\sum_{i=0}^{n-1} \min \left\{0, f\left(x_{i+1}\right)-f\left(x_{i}\right)\right\}, \\
& v_{f, P} \equiv \sum_{i=0}^{n-1}\left|f\left(x_{i+1}\right)-f\left(x_{i}\right)\right| .
\end{aligned}
$$

Denote by $T_{f}^{+}[0,1], T_{f}^{-}[0,1]$, and $T_{f}[0,1]$ the respective suprema of these quantities as $P$ ranges over all partitions of $[0,1]$, when the supremum exists; note that if $T_{f}[a, b]$ exists, then we say that $f$ has a variation and that $T_{f}[a, b]$ is the variation of $f$ on $[a, b]$. Applying Lemma 5 with

$$
d(x, y) \equiv \max \{0, f(x)-f(y)\} \quad(x, y \in[0,1])
$$

we see that if $T_{f}^{+}[0,1]$ exists, then so does $T_{f}^{+}[a, b]$ whenever $0 \leqslant a \leqslant b \leqslant 1$. Similar properties obtain for $T_{f}^{-}$and $T_{f}$. In the case of $T_{f}$, we recover a special case of Theorem 3 of [5]: if $f$ has a variation on $[0,1]$, then it has a variation on each compact subinterval of $[0,1]$. It is then straightforward to prove the additivity of the variation function:

$$
T_{f}[0,1]=T_{f}[0, c]+T_{f}[c, 1] \quad(0 \leqslant c \leqslant b)
$$

This brings us to our recursive example. 
Theorem 7 RUSS $\vdash$ There exists a Lipschitz mapping $f:[0,1] \rightarrow[0,1]$ that is not rectifiable.

Proof Let $\phi_{0}, \phi_{1}, \ldots$ be an effective enumeration of the computable partial functions in $\mathbf{N}^{\mathbf{N}}$, and for each $n$ define an increasing binary sequence $\left(a_{n, k}\right)_{k \geqslant 1}$ such that if $\phi_{n}(n)$ is computed in exactly $K$ steps, then $a_{n, k}=0$ for all $k<K$ and $a_{n, k}=1$ for all $k \geqslant K$. For each positive integer $n$ let

$$
J_{n} \equiv\left[\frac{1}{2^{n}}, \frac{1}{2^{n}}+\frac{1}{3^{n}}\right]
$$

Using Lemma 1, construct a function $g_{n}:\left[0,3^{-n}\right] \rightarrow\left[0,3^{-n}\right]$ such that

- $g_{n}(0)=g_{n}\left(3^{-n}\right)=0$,

- $g_{n}$ has Lipschitz constant 2,

- if $g_{n}$ is rectifiable, then either $a_{n, k}=0$ for each $k$ or else there exists $k$ such that $a_{n, k}=1$,

- if $g_{n}$ is differentiable at any point of $\left[0,3^{-n}\right]$, then either $a_{n, k}=0$ for each $k$ or else there exists $k$ such that $a_{n, k}=1$.

Now construct a uniformly continuous mapping $f_{n}:[0,1] \rightarrow\left[0,3^{-n}\right]$ such that

- $f_{n}(x)=0$ if either $0 \leqslant x \leqslant \frac{1}{2^{n}}$ or $\frac{1}{2^{n}}+\frac{1}{3^{n}} \leqslant x \leqslant 1$,

- $f_{n}(x)=g_{n}\left(x-\frac{1}{2^{n}}\right)$ if $\frac{1}{2^{n}} \leqslant x \leqslant \frac{1}{2^{n}}+\frac{1}{3^{n}}$,

- and $f_{n}$ has Lipschitz constant 2 .

The function $F \equiv \sum_{n=0}^{\infty} f_{n}$ is well defined and uniformly continuous on [0,1], since $\sum_{n=0}^{\infty}|| f_{n} \|$ converges by comparison with $\sum_{n=0}^{\infty} 3^{-2 n}$. Moreover, since the supports of the functions $f_{n}$ are pairwise apart, the restriction of $F$ to $J_{n}$ is $f_{n}$, and $F$ has Lipschitz constant 2. Suppose that (the graph of) $F$ is rectifiable. Then by Proposition 6, for each $n$ the restriction of $F$ to $J_{n}$ is rectifiable; whence $g_{n}$ is rectifiable, and therefore either $a_{n, k}=0$ for all $k$ or else $a_{n, k}=1$ for some $k$. It follows that the set

$$
K \equiv\left\{n \in \mathbf{N}: \phi_{n}(n) \text { is defined }\right\}
$$

is recursive, which is known to be false. Hence $F$ is not rectifiable over $[0,1]$.

Note that if the function $F$ constructed in the proof of Theorem 7 is differentiable at any point of $J_{n}$, then so is $g_{n}$, and we can decide whether $n \in K$. It follows that for each $n, F$ cannot be differentiable at any point of $J_{n}$. In light of this observation, if, in the proof of Theorem 7, we replace $J_{n}$ by $\left[\frac{1}{2^{n}}, \frac{1}{2^{n}}+\frac{2 \varepsilon}{3^{n}}\right]$, then we obtain the following: 
Corollary 8 RUSS $\vdash$ For each $\varepsilon>0$ there exist a Lipschitz function $f:[0,1] \rightarrow[0,1]$ and a set $J \subset[0,1]$ such that

(i) $f$ is not rectifiable;

(ii) $J$ is a union of countably many disjoint closed intervals of total length $\varepsilon$;

(iii) $f(x)=0$ for all $x \in[0,1]$ in the complement of $J$; and

(iv) $f$ is not differentiable at any point of $J$.

\section{Rectifiability and finite variation}

In this section we discuss the question: What, if any, is the connection between rectifiability and having a variation? First we dash any hope of proving that the latter property implies the former.

Proposition 9 For each binary sequence $\left(a_{n}\right)_{n \geqslant 1}$ there exists a function $g:[0,1] \rightarrow \mathbf{R}$ that has a variation but is rectifiable if and only if either $a_{n}=0$ for all $n$ or else there exists $n$ with $a_{n}=1$.

Proof Given a binary sequence $\left(a_{n}\right)_{n \geqslant 1}$, let $f:[0,1] \rightarrow \mathbf{R}$ be the function constructed in Lemma 1 above with $b=1$, and define $g:[0,1] \rightarrow \mathbf{R}$ by $g(x)=f(x) / 2+x$. Then $g$ is increasing: for if $0 \leqslant x_{1} \leqslant x_{2} \leqslant 1$, then

$$
\begin{aligned}
g\left(x_{2}\right)-g\left(x_{1}\right) & =\left(x_{2}-x_{1}\right)+\left(f\left(x_{2}\right)-f\left(x_{1}\right)\right) / 2 \\
& \geqslant\left(x_{2}-x_{1}\right)-\left|f\left(x_{2}\right)-f\left(x_{1}\right)\right| / 2 \geqslant 0 .
\end{aligned}
$$

Since $g$ is increasing, it has variation $g(1)-g(0)=1$. Now, if $a_{n}=0$ for all $n$, then $g(x)=x$ and $l_{g}=\sqrt{2}$. On the other hand, if $a_{n}=1$ for some $n$, then the slope of $g$ on the intervals $\left[k 2^{-n},\left(k+\frac{1}{2}\right) 2^{-n}\right]$ is

$$
1+\frac{1}{2} \frac{2^{n+1}}{2^{n}+1}=1+\frac{2^{n+1}}{2^{n+1}+2}
$$

and the slope of $g$ on the intervals $\left[\left(k+\frac{1}{2}\right) 2^{-n},(k+1) 2^{-n}\right]$ is

$$
1-\frac{2^{n+1}}{2^{n+1}+2} \text {. }
$$

Thus the arc length of $g$ over each $\left[k 2^{-n},(k+1) 2^{-n}\right]$ is

$$
2^{-(n+1)}\left(\sqrt{1+\left(1+\frac{2^{n+1}}{2^{n+1}+2}\right)^{2}}+\sqrt{1+\left(1-\frac{2^{n+1}}{2^{n+1}+2}\right)^{2}}\right)
$$


and since there are $2^{n}$ such intervals in question,

$$
\begin{aligned}
l_{g} & =\frac{1}{2} \sqrt{1+\left(1+\frac{2^{n+1}}{2^{n+1}+2}\right)^{2}}+\frac{1}{2} \sqrt{1+\left(1-\frac{2^{n+1}}{2^{n+1}+2}\right)^{2}} \\
& \geqslant \frac{\sqrt{34}+\sqrt{10}}{6} .
\end{aligned}
$$

Suppose that $g$ is rectifiable. Then either $l_{g}<(\sqrt{34}+\sqrt{10}) / 6$ or else $l_{g}>\sqrt{2}$. In the first case there can be no $n$ with $a_{n}=1$, so $a_{n}=0$ for all $n$. In the second case, taking a partition $P$ of $[0,1]$ such that $l_{g, P}>\sqrt{2}$, we can find $x \in P$ such that $g(x) \neq x$. Let $N$ be such that $\sum_{n=N}^{\infty}|| f_{n}||<|g(x)-x|$, where $\left(f_{n}\right)_{n \geqslant 1}$ is the sequence in Lemma 1 with $f=\sum_{n=1}^{\infty} f_{n}$. Then there exists $n<N$ such that $a_{n}=1$, for if not

$$
|g(x)-x|=|f(x)| \leqslant \sum_{n=1}^{\infty}|| f_{n}||=\sum_{n=N}^{\infty}|| f_{n}||<|g(x)-x|,
$$

which is absurd.

Corollary 10 The statement 'Every real-valued Lipschitz function $f:[0,1] \rightarrow \mathbf{R}$ with a variation is rectifiable' implies LPO.

Corollary 11 If the set of real-valued functions on $[0,1]$ that have a variation is closed under addition, then LPO is derivable.

Proof With $f, g$, and $\left(a_{n}\right)_{n \geqslant 1}$ as in the proof of Proposition 9, both $g$ and the identity function id on $[0,1]$ have a variation, but if $f=\mathrm{id}+g$ has a variation, then either $a_{n}=0$ for all $n$ or there exists $n$ with $a_{n}=1$.

In contrast to Corollary 11, we have:

Proposition 12 The set of real valued, rectifiable functions on $[0,1]$ is closed under addition.

Proof This is a simple application of the triangle inequality.

Corollary 10 shows that we cannot prove constructively that every Lipschitz, let alone every continuous, function $f:[0,1] \rightarrow \mathbf{R}$ with a variation is rectifiable. Our final task is to show that, in contrast, we can prove that every rectifiable continuous mapping $f:[0,1] \rightarrow \mathbf{R}$ has a variation. This will require some preliminaries. 
Lemma 13 Let $a, b, c$ be nonnegative numbers. Then

$$
\sqrt{a^{2}+(b+c)^{2}}-\sqrt{a^{2}+b^{2}} \geqslant \sqrt{a^{2}+c^{2}}-a .
$$

Proof First take the case $a=1$. Define

$$
f(x)=\sqrt{1+(x+c)^{2}}-\sqrt{1+x^{2}} \quad(x \geqslant 0) .
$$

Then (we omit the details) $f^{\prime} \geqslant 0$, which ensures that

$$
f(x) \geqslant f(0)=\sqrt{1+c^{2}}-1 \quad(x \geqslant 0) .
$$

Next take the case $a>0$. By the first case,

$$
\begin{aligned}
\sqrt{a^{2}+(b+c)^{2}}-\sqrt{a^{2}+b^{2}} & =a\left(\sqrt{1+\left(\frac{b}{a}+\frac{c}{a}\right)^{2}}-\sqrt{1+\left(\frac{b}{a}\right)^{2}}\right) \\
& \geqslant a\left(\sqrt{1+\left(\frac{c}{a}\right)^{2}}-1\right)=\sqrt{a^{2}+c^{2}}-a .
\end{aligned}
$$

Finally, if $a \geqslant 0$, then for each $\varepsilon>0$ we have $a+\varepsilon>0$, so

$$
\sqrt{(a+\varepsilon)^{2}+(b+c)^{2}}-\sqrt{(a+\varepsilon)^{2}+b^{2}} \geqslant \sqrt{(a+\varepsilon)^{2}+c^{2}}-(a+\varepsilon) .
$$

Letting $\varepsilon \rightarrow 0$ completes the proof.

Lemma 14 Let $0=x_{0}<x_{1}<\cdots<x_{m}=1$ and let $\varepsilon_{0}, \varepsilon_{1}, \ldots, \varepsilon_{m-1}$ be nonnegative numbers. Then

$$
\sum_{i=0}^{m-1} \sqrt{\left(x_{i+1}-x_{i}\right)^{2}+\varepsilon_{i}^{2}} \geqslant \sqrt{1+\sum_{i=0}^{m-1} \varepsilon_{i}^{2}}
$$

Proof Denoting by $\rho$ the Euclidean distance function on $\mathbf{R}^{2}$, first note that

$$
\sqrt{\left(x_{1}-x_{0}\right)^{2}+\varepsilon_{0}^{2}}=\rho\left(\left(x_{0}, 0\right),\left(x_{1}, \varepsilon_{0}\right)\right),
$$

and for $i \geqslant 1$,

$$
\begin{aligned}
\sqrt{\left(x_{i+1}-x_{i}\right)^{2}+\varepsilon_{i}^{2}} & =\sqrt{\left(x_{i+1}-x_{i}\right)^{2}+\left(\left(\varepsilon_{i-1}+\varepsilon_{i}\right)-\varepsilon_{i-1}\right)^{2}} \\
& =\rho\left(\left(x_{i}, \varepsilon_{i-1}\right),\left(x_{i+1}, \varepsilon_{i-1}+\varepsilon_{i}\right)\right) .
\end{aligned}
$$

With $\epsilon_{-1}=0$, an induction now shows that for $k<m$,

$$
\sum_{i=0}^{k} \sqrt{\left(x_{i+1}-x_{i}\right)^{2}+\varepsilon_{i}^{2}}=\sum_{i=0}^{k} \rho\left(\left(x_{i}, \varepsilon_{0}+\cdots+\varepsilon_{i-1}\right),\left(x_{i+1}, \varepsilon_{0}+\cdots+\varepsilon_{i}\right)\right) .
$$


Thus

$$
\sum_{i=0}^{m-1} \sqrt{\left(x_{i+1}-x_{i}\right)^{2}+\varepsilon_{i}^{2}}=\sum_{i=0}^{m-1} \rho\left(\left(x_{i}, \varepsilon_{0}+\cdots+\varepsilon_{i-1}\right),\left(x_{i+1}, \varepsilon_{0}+\cdots+\varepsilon_{i}\right)\right),
$$

which is at least the straight-line distance from $\left(x_{0}, \varepsilon_{-1}\right)$ to $\left(x_{m}, \varepsilon_{0}+\cdots+\varepsilon_{m-1}\right)$. Hence

$$
\begin{aligned}
\sum_{i=0}^{m-1} \sqrt{\left(x_{i+1}-x_{i}\right)^{2}+\varepsilon_{i}^{2}} & \geqslant \sqrt{\left(x_{m}-x_{0}\right)^{2}+\left(\varepsilon_{0}+\cdots+\varepsilon_{m-1}\right)^{2}} \\
& =\sqrt{1+\left(\sum_{i=0}^{m-1} \varepsilon_{i}\right)^{2}}
\end{aligned}
$$

Lemma 15 Let $f:[0,1] \rightarrow \mathbf{R}$ be continuous. Let $\varepsilon>0$, and let $P, P^{\prime}$ be partitions of $[0,1]$ such that $P \subset P^{\prime}$ and $v_{f, P^{\prime}}-v_{f, P}>\varepsilon$. Then

$$
l_{f, P^{\prime}}-l_{f, P}>\sqrt{1+\varepsilon^{2}}-1 \text {. }
$$

Proof To begin with, consider the case where

(i) $P$ is the trivial partition $\{0,1\}$ and

(ii) $P^{\prime}: 0=x_{0}<x_{1}<\cdots<x_{n}=1$ is a strict partition with $f\left(x_{i}\right) \neq f\left(x_{j}\right)$ whenever $0 \leqslant i<j<n$.

In view of (ii), the function $s: n \rightarrow\{-1,1\}$ given by

$$
s(i)=\left\{\begin{aligned}
-1 & \text { if } f\left(x_{i+1}\right)-f\left(x_{i}\right)<0 \\
1 & \text { if } f\left(x_{i+1}\right)-f\left(x_{i}\right)>0
\end{aligned}\right.
$$

is well defined. Construct a piecewise-linear function $g:[0,1] \rightarrow \mathbf{R}$ such that $g(0)=0$ and

$$
g\left(x_{i}\right)=\sum_{j \in i} s(j)\left(f\left(x_{j+1}\right)-f\left(x_{j}\right)\right) \quad(1 \leqslant i \leqslant n) .
$$

Thus $g(0)=0$ and

$$
\begin{aligned}
g\left(x_{1}\right) & =s(1)\left(f\left(x_{1}\right)-f\left(x_{0}\right)\right), \\
g\left(x_{2}\right) & =s(1)\left(f\left(x_{1}\right)-f\left(x_{0}\right)\right)+s(2)\left(f\left(x_{2}\right)-f\left(x_{0} 1\right),\right. \\
& \vdots \\
g(1) & =\sum_{j=0}^{n-1} s(j)\left(f\left(x_{j+1}\right)-f\left(x_{j}\right)\right) .
\end{aligned}
$$


Note that if $x \in\left[x_{j}, x_{j+1}\right]$ and $y \in\left[x_{j+1}, x_{j+2}\right]$, then

$$
g(y)=g(x)+s(j)\left(f\left(x_{j+2}\right)-f\left(x_{j+1}\right)\right)>g(x) ;
$$

whence $g$ is monotone and so has variation $g(1)-g(0)$. Moreover, for each $j$,

$$
g\left(x_{j+1}\right)-g\left(x_{j}\right)=s(j)\left(f\left(x_{j+1}\right)-f\left(x_{j}\right)\right)
$$

and therefore the variation of $g$ on $\left[x_{j}, x_{j+1}\right]$ is $\left|f\left(x_{j+1}\right)-f\left(x_{j}\right)\right|$. Since the variation function is additive, it follows that

$$
v_{f, P^{\prime}}=\sum_{j=0}^{n-1}\left|f\left(x_{j+1}\right)-f\left(x_{j}\right)\right|=v_{g, P^{\prime}}=g(1)-g(0) .
$$

It follows also from (2) that $l_{f, P^{\prime}}=l_{g, P^{\prime}}$. Since adding points to a partition cannot decrease the approximations to the length of the curves of $f$ or $g$, we now see that

$$
\begin{aligned}
l_{f, P^{\prime}}=l_{g, P^{\prime}} & \geqslant \sqrt{1+(g(1)-g(0))^{2}} \\
& =\sqrt{1+v_{f, P^{\prime}}^{2}} \geqslant \sqrt{1+\left(v_{f, P}+\varepsilon\right)^{2}}
\end{aligned}
$$

and hence, via Lemma 13, that

$$
l_{f, P^{\prime}}-l_{f, P} \geqslant \sqrt{1+\left(v_{f, P}+\varepsilon\right)^{2}}-\sqrt{1+v_{f, P}^{2}} \geqslant \sqrt{1+\varepsilon^{2}}-1 .
$$

We now weaken the assumption (i) by taking $P$ as a strict partition $0<\xi_{1}<\xi_{2}<$ $\cdots<\xi_{m}=1$. For each $i$ let

$$
P_{i}=\left\{x_{i}, x_{i+1}\right\}, P_{i}^{\prime}=\left\{x_{j}: \xi_{i} \leqslant x_{j} \leqslant \xi_{i+1}\right\}, \text { and } g_{i}=\left.f\right|_{\left[\xi_{i}, \xi_{i+1}\right]} .
$$

Note that $P_{i} \subset P_{i}^{\prime}$, that

$$
v_{f, P^{\prime}}-v_{f, P}=\sum_{i=0}^{m-1}\left(v_{f, P_{i}^{\prime}}-v_{f, P_{i}}\right)
$$

and that

$$
l_{f, P^{\prime}}-l_{f, P}=\sum_{i=0}^{m-1}\left(l_{f, P_{i}^{\prime}}-l_{f, P_{i}}\right)
$$

For $0 \leqslant i<m$ let

$$
\varepsilon_{i}=v_{f, P_{i}^{\prime}}-v_{f, P_{i}} \geqslant 0 .
$$

Note that since the variation function is additive,

$$
\sum_{i=0}^{m-1} \varepsilon_{i}=\sum_{i=0}^{m-1} v_{f, P_{i}^{\prime}}-\sum_{i=0}^{m-1} v_{f, P_{i}}=v_{f, P^{\prime}}-v_{f, P}>\varepsilon .
$$


Now, by the first part of the proof,

$$
\begin{aligned}
l_{f, P_{i}^{\prime}}-l_{f, P_{i}} & \geqslant \sqrt{\left(x_{i+1}-x_{i}\right)^{2}+v_{f, P_{i}^{\prime}}^{2}}-\sqrt{\left(x_{i+1}-x_{i}\right)^{2}+v_{f, P_{i}}^{2}} \\
& =\sqrt{\left(x_{i+1}-x_{i}\right)^{2}+\left(v_{f, P_{i}}+\varepsilon_{i}\right)^{2}}-\sqrt{\left(x_{i+1}-x_{i}\right)^{2}+v_{f, P_{i}}^{2}} \\
& \geqslant \sqrt{\left(x_{i+1}-x_{i}\right)^{2}+\varepsilon_{i}^{2}}-\left(x_{i+1}-x_{i}\right) .
\end{aligned}
$$

Since length is additive, we obtain

$$
\begin{aligned}
l_{f}\left(P^{\prime}\right)-l_{f}(P) & =\sum_{i=0}^{m-1}\left(l_{f, P_{i}^{\prime}}-l_{f, P_{i}}\right) \\
& \geqslant \sum_{i=0}^{m-1}\left(\sqrt{\left(x_{i+1}-x_{i}\right)^{2}+\varepsilon_{i}^{2}}-\left(x_{i+1}-x_{i}\right)\right) \\
& =\sum_{i=0}^{m-1} \sqrt{\left(x_{i+1}-x_{i}\right)^{2}+\varepsilon_{i}^{2}}-\sum_{i=0}^{m-1}\left(x_{i+1}-x_{i}\right) \\
& =\sum_{i=0}^{m-1} \sqrt{\left(x_{i+1}-x_{i}\right)^{2}+\varepsilon_{i}^{2}}-1 \\
& \geqslant \sqrt{1+\left(\sum_{i=0}^{m-1} \varepsilon_{i}\right)^{2}}-1
\end{aligned}
$$

the last step coming from Lemma 14. Taken with (3), this leads us to the desired conclusion in the case under consideration. To complete the proof in the general case, we need only use a simple continuity argument.

Theorem 16 If $f:[0,1] \rightarrow \mathbf{R}$ is continuous and rectifiable, then it has a variation.

Proof Let

$$
S \equiv\left\{v_{f, P}: P \text { is a partition of }[0,1]\right\} .
$$

Given real numbers $\alpha, \beta$ with $\alpha<\beta$, let $\varepsilon=\frac{\alpha+\beta}{2}$ and let $P$ be a partition of $[0,1]$ such that

$$
l_{f}-l_{f, P}<\sqrt{1+\varepsilon^{2}}-1 .
$$

Either $v_{f, P}>\alpha$ or $v_{f, P}<(\beta-\alpha) / 2$. In the latter case, consider any partition $P^{\prime}$ of $[0,1]$. By Lemma 15,

$$
v_{f, P \cup P^{\prime}} \leqslant v_{f, P}+\varepsilon<\beta .
$$


It follows from the constructive least-upper-bound principle that the variation of $f$ on $[0,1]$ exists.

\section{Concluding remarks}

In view of Lemma 1, we might ask for Brouwerian counterexamples to such statements as these:

- Every real-valued function on $[0,1]$ whose derivative exists at each point is rectifiable.

- Every real-valued Lipschitz function on $[0,1]$ that is differentiable almost everywhere is rectifiable.

There can be no Brouwerian counterexamples for these two statements, since each of them is provable in INT. If $f:[0 ; 1] \rightarrow \mathbf{R}$ is differentiable everywhere, then intuitionistically its derivative $f^{\prime}$ is not just continuous, but uniformly continuous, so we can rectify the graph of $f$ by the usual calculus formula for arc length. On the other hand, if $f$ has Lipschitz constant $c>0$ and is pointwise differentiable almost everywhere, then $\left|f^{\prime}(x)\right| \leqslant c$ at any point $x$ where $f$ is differentiable. A theorem of van Rootselaar (see Heyting [11, page 79] or Bridges and Demuth [7, Theorem 6]) shows that $f^{\prime}$ is intuitionistically measurable, whence by Bishop and Bridges [4, Theorem (7.11), page 263] it is integrable. We can then show that the supremum of the set

$$
\left\{\sum_{i=0}^{n-1} \sqrt{\left(x_{i+1}-x_{i}\right)^{2}+\left(f\left(x_{i+1}\right)-f\left(x_{i}\right)\right)^{2}}: P \text { is a partition of }[0,1]\right\}
$$

exists and equals

$$
\int_{0}^{1}\left(1+f^{\prime 2}\right)^{1 / 2} d x
$$

It is possible that there is a recursive example of a real-valued Lipschitz function on $[0,1]$ that is differentiable almost everywhere but not rectifiable; but we do not know of one.

\section{References}

[1] P. Aczel and M. Rathjen, Notes on Constructive Set Theory, Report No. 40, Institut Mittag-Leffler, Royal Swedish Academy of Sciences, 2001. 
[2] P. Aczel and M. Rathjen: Constructive Set Theory, monograph, forthcoming; preprint available at http://www1.maths.leeds.ac.uk//rathjen/preprints.html

[3] E. Bishop: Foundations of Constructive Analysis, McGraw-Hill, New York, 1967.

[4] E. Bishop and D.S. Bridges: Constructive Analysis, Grundlehren der math. Wissenschaften 279, Springer Verlag, Heidelberg, 1985.

[5] D.S. Bridges: 'A constructive look at functions of bounded variation', Bull. London Math. Soc. 32(3), 316-324, 2000; doi: 10.1112/s0024609399006736

[6] D.S. Bridges and R.A. Alps, Morse Set Theory as a Foundation for Constructive Mathematics, monograph in preparation, University of Canterbury, Christchurch, New Zealand, 2017.

[7] D. Bridges and O. Demuth, 'On the Lebesgue measurability of continuous functions in constructive analysis', Bull. Amer. Math. Soc. 24(2), 259-276, 1991; doi: 10.1090/s0273-0979-1991-16014-3

[8] D.S. Bridges and F. Richman: Varieties of Constructive Mathematics, London Math. Soc. Lecture Notes 97, Cambridge Univ. Press, 1987; doi: 10.1017/CBO9780511565663

[9] D.S. Bridges and L.S. Vîţă: Techniques of Constructive Analysis, Universitext, Springer New York, 2006; doi: 10.1007/978-0-387-38147-3

[10] M.A.E. Dummett, Elements of Intuitionism, Oxford Logic Guides 39, Clarendon Press, Oxford, 2000.

[11] A. Heyting, Intuitionism, Third Edition, North-Holland Publ. Co., Amsterdam, 1971.

[12] B.A. Kushner, Lectures on Constructive Mathematical Analysis, Amer. Math. Soc., Providence RI, 1985.

[13] A.A. Markov, Theory of Algorithms (Russian), Trudy Mat. Istituta imeni V.A. Steklova 42 (Izdatel'stvo Akademii Nauk SSSR, Moskva), 1954.

[14] P. Martin-Löf: 'An Intuitionistic Theory of Types: Predicative Part', in: Logic Colloquium 1973 (H.E. Rose and J.C. Shepherdson, eds), 73-118, North-Holland, Amsterdam, 1975; doi: 10.1016/s0049-237x(08)71945-1

[15] P. Martin-Löf: Intuitionistic type theory (Notes by Giovanni Sambin of a series of lectures given in Padua, June 1980), Bibliopolis, Napoli,1984.

[16] B. Nordström, K. Peterson, and J.M. Smith: Programming in Martin-Löf's Type Theory. Oxford University Press, 1990. (Also available at http://www .cse.chalmers . se/ research/group/logic/book/)

[17] E. Specker, 'Nicht konstruktiv beweisbare Sätze der Analysis', J. Symbolic Logic 14, 145-158, 1949; doi: 10.2307/2267043

[18] A.S. Troelstra and D van. Dalen: Constructivism in Mathematics (Vol. I), Studies in Logic and the Foundations of Mathematics 121, North-Holland Publishing Co., Amsterdam, 1988; doi: 10.1016/s0049-237x(09)70525-7 
School of Mathematics \& Statistics, University of Canterbury

Private Bag 4800, Christchurch 8140, New Zealand

School of Mathematics \& Statistics, University of Canterbury

Private Bag 4800, Christchurch 8140, New Zealand

Department of Mathematics, Stockholm University

SE-106 91, Stockholm, Sweden

d.bridges@math.canterbury.ac.nz, matthew.hendtlass@canterbury.ac.nz, palmgren@math.su.se

Received: 24 September 2015 Revised: 31 December 2016 